\title{
Engineering superfluidity in Bose-Fermi mixtures of ultracold atoms
}

\section{Citation}

Wang, D.-W., M. D. Lukin, and E. Demler. 2005. "Engineering Superfluidity in Bose-

Fermi Mixtures of Ultracold Atoms." Physical Review A 72 (5) (November 17). doi:10.1103/ physreva.72.051604.

\section{Published Version}

doi:10.1103/physreva.72.051604

\section{Permanent link}

http://nrs.harvard.edu/urn-3:HUL.InstRepos:27899421

\section{Terms of Use}

This article was downloaded from Harvard University's DASH repository, and is made available under the terms and conditions applicable to Other Posted Material, as set forth at http:// nrs.harvard.edu/urn-3:HUL.InstRepos:dash.current.terms-of-use\#LAA

\section{Share Your Story}

The Harvard community has made this article openly available.

Please share how this access benefits you. Submit a story.

Accessibility 


\title{
Engineering superfluidity in Bose-Fermi mixtures of ultracold atoms
}

\author{
D.-W. Wang, ${ }^{1}$ M. D. Lukin, ${ }^{2}$ and E. Demler ${ }^{2}$ \\ ${ }^{1}$ Department of Physics, National Tsing-Hua University, Hsinchu, Taiwan 300, Republic of China \\ ${ }^{2}$ Physics Department, Harvard University, Cambridge, Massachusetts 02138, USA
}

(Received 19 October 2004; published 17 November 2005)

\begin{abstract}
We investigate many-body phase diagrams of atomic boson-fermion mixtures loaded in the two-dimensional optical lattice. Bosons mediate an attractive, finite-range interaction between fermions, leading to fermion pairing phases of different orbital symmetries. Specifically, we show that by properly tuning atomic and lattice parameters it is possible to create superfluids with $s-, p$-, and $d$-wave pairing symmetry as well as spin and charge density wave phases. These phases and their stability are analyzed within the mean-field approximation for systems of ${ }^{40} \mathrm{~K}-{ }^{87} \mathrm{Rb}$ and ${ }^{40} \mathrm{~K}-{ }^{23} \mathrm{Na}$ mixtures. For the experimentally accessible regime of parameters, superfluids with unconventional fermion pairing have transition temperature around a percent of the Fermi energy.

DOI: 10.1103/PhysRevA.72.051604

PACS number(s): 03.75.Mn, 03.75.Hh, 74.20.Fg, 74.20.Rp
\end{abstract}

Mixtures of quantum degenerate atoms recently became a subject of intense studies. Examples include recent experimental observations of instabilities in Bose-Fermi mixtures [1], superfluidity of fermion pairing [2], and condensation of molecules in fermionic mixtures [3]. Many other intriguing many-body effects have been proposed theoretically. They include formation of composite particles [4], appearance of charge density wave order [5], phonon-induced fermion pairing $[6,7]$, and polaronic effects [8]. In this paper, we study quantum phases of boson-fermion mixtures (BFM) in twodimensional (2D) optical lattices. The fermionic atoms are prepared as a mixture of two hyperfine spin states, which interact via short-range repulsive interaction. Density fluctuations in a condensate of bosonic atoms induce an attractive interaction between fermions, which is of finite range. Competition between these two types of interactions results in several many-body phases of fermions by appropriately choosing atomic and lattice parameters. These include charge and spin density wave phases (CDW/SDW) as well as superfluid states with unconventional pairing of fermions. We discuss how these phases can be observed in realistic parameter regime of interest [9]. Experimental realization of such systems should provide critical insights into understanding several important strongly correlated electron systems, including quasi-2D unconventional superconductors, such as high $T_{c}$ cuprates [10] and organic conductors [11] displaying $d$-wave superconductivity, as well as ruthenates [12] and Bechgaard salts [11] displaying $p$-wave superconductivity.

We first describe the microscopic theory for the BFM systems in 2D optical lattice. When the lattice potential is strong enough, the BFM system can be described by the single band Hubbard type Hamiltonian $[8,13]$

$$
\begin{aligned}
H= & \sum_{\mathbf{k}}\left[\bar{\epsilon}_{\mathbf{k}}^{b} \hat{b}_{\mathbf{k}}^{\dagger} \hat{b}_{\mathbf{k}}+\bar{\epsilon}_{\mathbf{k}, \uparrow}^{f} \hat{f}_{\mathbf{k}, \uparrow}^{\dagger} \hat{f}_{\mathbf{k}, \uparrow}+\bar{\epsilon}_{\mathbf{k}, \downarrow}^{f} \hat{f}_{\mathbf{k}, \downarrow}^{\dagger} \hat{f}_{\mathbf{k}, \downarrow}\right] \\
& +\frac{1}{\Omega} \sum_{\mathbf{k}}\left[\frac{U_{b b}}{2} \hat{\rho}_{\mathbf{k}}^{b} \hat{\rho}_{-\mathbf{k}}^{b}+U_{b f} \hat{\rho}_{\mathbf{k}}^{b} \hat{\rho}_{-\mathbf{k}}^{f}+U_{f f} \hat{\rho}_{\mathbf{k}, \uparrow}^{f} \hat{\rho}_{-\mathbf{k}, \downarrow}^{f}\right]
\end{aligned}
$$

where $\hat{b}_{\mathbf{k}}$ and $\hat{f}_{\mathbf{k}, s}$ are the annihilation operators for bosonic and fermionic atoms with momentum k. $\hat{\rho}_{\mathbf{k}}^{b}=\sum_{\mathbf{p}} \hat{b}_{\mathbf{p}+\mathbf{k}}^{\dagger} \hat{b}_{\mathbf{p}}$ is the boson density operators, and $\bar{\epsilon}_{\mathbf{k}}^{b} \equiv \epsilon_{\mathbf{k}}^{b}-\mu_{b}$, where $\epsilon_{\mathbf{k}}^{b}=-t_{b} \gamma_{\mathbf{k}}$ is the single particle energy with $t_{b}$ being the tunneling amplitude of bosons between neighboring sites and $\gamma_{\mathbf{k}} \equiv 2\left(\cos k_{x}+\cos k_{y}\right)$ (lattice constant is set to be unit). $\mu_{b}$ is the boson chemical potential. Similar notations also apply to fermions with superscript $f$ and $\hat{\rho}_{\mathbf{k}}^{f} \equiv \hat{\rho}_{\mathbf{k}, \uparrow}^{f}+\hat{\rho}_{\mathbf{k}, \downarrow}^{f}$ in Eq. (1). $U_{b b}, U_{b f}$, and $U_{f f}$ are, respectively, boson-boson, bosonfermion, and fermion-fermion onsite interaction energy, which can be calculated from the $s$-wave scattering length and the lattice potential $[13,14] . \Omega$ is the system volume. For simplicity we neglect the global trapping potential and consider systems with uniform densities.

We are interested in the low temperature regime where the bosonic atoms are condensed $\left(\mu_{b}=\epsilon_{0}^{b}\right)$. Using Bogoliubov approximation, one can obtain an effective fermionphonon coupling Hamiltonian [6,15]. The phonon field can be integrated out [6] to provide an effective attractive interaction between fermion atoms and hence cause the fermion pairing. If the phonon velocity $c$ is much larger than the Fermi velocity $v_{f}$ (i.e., in the fast phonon limit), the resulting interaction between fermions is instantaneous and given by $V_{\text {ind }}(\mathbf{k})=-\tilde{V} /\left[1+\xi^{2}\left(4-\gamma_{\mathbf{k}}\right)\right]$, where $\tilde{V} \equiv U_{b f}^{2} / U_{b}$ is the strength of the phonon-induced attractive interaction and $\xi=\sqrt{t_{b} / 2 n_{b} U_{b b}}$ is the boson correlation (healing) length. Such antiadiabatic limit may not be easily achieved in typical ${ }^{40} \mathrm{~K}-{ }^{87} \mathrm{Rb}$ system $\left(c / v_{f} \sim 1\right)$ because the boson atom mass is larger than the fermion atoms. In this paper, therefore, we also consider a BFM system composed by ${ }^{40} \mathrm{~K}$ and ${ }^{23} \mathrm{Na}$ atoms, where the phone velocity can be several times larger than the Fermi velocity $\left(c / v_{f} \sim 5\right)$. We note that including the retardation effects just changes the prefactor in the BCS expression for $T_{c}$ from the Fermi energy $E_{f}$ to some characteristic bosonic frequency [16], and therefore it should provide similar quantum phases as obtained within the fast phonon limit (see also Refs. [6,7]). As a result, we may still apply the fast phonon approximation and obtain the following effective fermion Hamiltonian:

$$
H_{\mathrm{eff}}=\sum_{\mathbf{k}, s} \bar{\epsilon}_{\mathbf{k}}^{f} \hat{f}_{\mathbf{k}, s}^{\dagger} \hat{f}_{\mathbf{k}, s}+\frac{1}{2 \Omega} \sum_{\mathbf{k}, s, s^{\prime}} V_{\mathrm{eff}}^{s, s^{\prime}} \hat{\rho}_{\mathbf{k}, s} \hat{\rho}_{-\mathbf{k}, s^{\prime}},
$$

where $V_{\mathrm{eff}}^{s, s^{\prime}}(\mathbf{k}) \equiv U_{f f} \delta_{s,-s^{\prime}}+V_{\text {ind }}(\mathbf{k})$. 
Following the early work of Micnas et al. [17], we apply the mean-field approximation to calculate the $T_{c}$ of fermion pairing phases and that of the competing SDW/CDW phases. For the superfluid states, the single particle excitation energy $E_{\mathbf{k}}$ has a gap at Fermi surface: $E_{\mathbf{k}}=\sqrt{\left(\bar{\epsilon}_{\mathbf{k}}^{f}+\Sigma_{\mathbf{k}}\right)^{2}+\left|\Delta_{\mathbf{k}}^{s, s^{\prime}}\right|^{2}}$, where the gap function, $\Delta_{\mathbf{k}}^{s, s^{\prime}} \equiv \Omega^{-1} \Sigma_{\mathbf{p}} V_{\mathrm{eff}}^{s, s^{\prime}}(\mathbf{k}-\mathbf{p})\left\langle\hat{f}_{\mathbf{p}, s}^{\dagger} \hat{f}_{-\mathbf{p}, s^{\prime}}^{\dagger}\right\rangle$, is determined by the gap equation

$$
\Delta_{\mathbf{k}}^{s, s^{\prime}}=\frac{-1}{2 \Omega} \sum_{\mathbf{p}} V_{\mathrm{eff}}^{s, s^{\prime}}(\mathbf{k}-\mathbf{p}) \frac{\Delta_{\mathbf{p}}^{s, s^{\prime}}}{E_{\mathbf{p}}} \tanh \left(\frac{E_{\mathbf{p}}}{2 T}\right) .
$$

Here $\Sigma_{\mathbf{k}}=-(1 / \Omega) \Sigma_{\mathbf{p}} V_{\text {eff }}^{s, s}(\mathbf{k}-\mathbf{p})\left\langle\hat{f}_{\mathbf{p}, \hat{s}}^{\dagger} \hat{f}_{\mathbf{p}, s}\right\rangle$ is fermion exchange self-energy within Hartree-Fock (HF) approximation. Finally the fermion chemical potential (corrected by the Hartree energy) is fixed by the known total density of fermions

$$
n_{f}=\frac{1}{\Omega} \sum_{\mathbf{p}, s}\left\langle\hat{f}_{\mathbf{p}, s}^{\dagger} \hat{f}_{\mathbf{p}, s}\right\rangle=1-\frac{1}{\Omega} \sum_{\mathbf{p}} \frac{\bar{\epsilon}_{\mathbf{p}}^{f}}{E_{\mathbf{p}}} \tanh \left(\frac{E_{\mathbf{p}}}{2 T}\right) .
$$

To analyze the results of different gap symmetries, we consider the following ansatz for the gap function [17]: $\Delta_{\mathbf{k}}^{s, s^{\prime}}=\delta_{s,-s^{\prime}}\left(\Delta_{s 0}+\Delta_{s 1} \gamma_{\mathbf{k}}+\Delta_{d} \eta_{\mathbf{k}}\right)+\delta_{s, s^{\prime}} \Delta_{p} \sin k_{x}$, where $\eta_{\mathbf{k}} \equiv 2\left(\cos k_{x}-\cos k_{y}\right)$. Here $\Delta_{s 0}$ and $\Delta_{s 1}$ are for the onsite and extended $s$-wave pairing phase, while $\Delta_{p / d}$ is for the $p$-/d-wave pairing phase. The transition temperature $T_{c}$ is then numerically solved by setting $\Delta_{s 0, s 1, d, p}^{s, s^{\prime}} \rightarrow 0$ in Eqs. (3) and (4). A similar approach can also be used to analyze the SDW/CDW phases [18], where the associated order parameters are, respectively, the spin and charge density components at certain wave-vector $\mathbf{Q}$ (i.e. $\Sigma_{\mathbf{k}}\left\langle f_{\mathbf{k}+\mathbf{Q}, \uparrow}^{\dagger} f_{\mathbf{k}, \downarrow}\right\rangle \neq 0$ for SDW and $\Sigma_{\mathbf{k}, s}\left\langle f_{\mathbf{k}+\mathbf{Q}, s}^{\dagger} f_{\mathbf{k}, s}\right\rangle \neq 0$ for CDW). Here $\mathbf{Q}=(\pi, \pi)$ is the 2D nesting wave vector at half-filling (we set the lattice constant $a$ to be unit). In such a case, the SDW/CDW phase shows the periodic modulation of pseudospin and atomic densities, respectively, in the 2D optical lattice with period $2 a$. Away from half-filling, the SDW/CDW phases for the commensurate wave-vector $\mathbf{Q}=(\pi, \pi)$ may be less favorable than density wave phases at incommensurate wavevectors [19] (i.e., the density oscillation period is not a multiple of the lattice constant). The later, however, are much more difficult to analyze than their commensurate analogues. In this paper we therefore discuss only the commensurate SDW/ CDW phases, but we believe they provide an accurate estimate for the regime of more general SDW/CDW phases.

Finally we study the stability of the many-body phases discussed above. The mean field (MF) ground state energy of a pairing phase can be derived to be

$$
\begin{aligned}
E_{\mathrm{MF}}= & \sum_{\mathbf{k}}\left(\bar{\epsilon}_{\mathbf{k}}^{f}+\Sigma_{\mathbf{k}}-E_{\mathbf{k}}\right)+\mu_{f} N_{f} \\
& +\frac{N_{f}^{2}}{4 \Omega}\left[U_{f f}+2 V_{\mathrm{ph}}(0)\right]+\sum_{\mathbf{k}, s, s^{\prime}} \frac{\left|\Delta_{\mathbf{k}}^{s, s^{\prime}}\right|^{2}}{4 E_{\mathbf{k}}},
\end{aligned}
$$

where $\mu_{f}$ is determined via Eq. (4) by conserving the total number of fermions $N_{f}$. The stability condition results from requiring that the compressibility (or bulk modulus $B)$ be positive. At zero temperature it is defined by

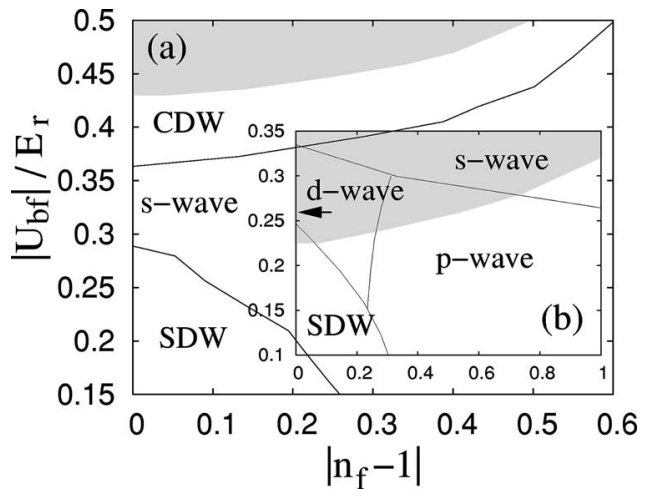

FIG. 1. (a) Phase diagrams of ${ }^{40} \mathrm{~K}-{ }^{87} \mathrm{Rb}$ mixtures (system A) and (b) ${ }^{40} \mathrm{~K}-{ }^{23} \mathrm{Na}$ mixtures (system B) loaded in $2 \mathrm{D}$ optical lattice with Nd:YAG laser wavelength $\lambda=1.06 \mu \mathrm{m} .{ }^{40} \mathrm{~K}$ are prepared in the hyperfine states $\left|F, m_{F}\right\rangle=|9 / 2,-9 / 2\rangle$ and $|9 / 2,-7 / 2\rangle$ corresponding to the pseudospin up and down fermions. Bosonic atoms $\left({ }^{87} \mathrm{Rb}\right.$ and $\left.{ }^{23} \mathrm{Na}\right)$ are in state $|1,1\rangle . n_{f}$ is fermion filling fraction and the in-plane lattice potential is chosen to be $V_{0, \|}=5 E_{r}$ and it is $V_{0, z}=30 E_{r}$ in the $z$ direction to form a (quasi-)2D system. $E_{r}=4.44 \mathrm{kHz}$ is the recoil energy of ${ }^{40} \mathrm{~K}$. We choose boson filling fraction $n_{b}=1$ for system A but $n_{b}=9$ for system B, respectively. The shaded areas are the regimes of collapse. Arrows in (b) indicate the background interaction strength (Ref. [14]), while it is at $0.84 E_{r}$ and beyond the scale of (a). From (b), the $d$-wave pairing phase should exist near half-filling with $a_{b f} \sim-100 a_{0}$ (Ref. [14]).

$B \equiv-\Omega(\partial P / \partial \Omega)_{N_{f}}=n_{f}^{2} \partial^{2} \mathcal{E}_{M F} / \partial n_{f}^{2}$, where $P \equiv-\left(\partial E_{\mathrm{MF}} / \partial \Omega\right)_{N_{f}}$ is the pressure of the atomic gas and $\mathcal{E}_{\mathrm{MF}}=E_{\mathrm{MF}} / \Omega$ is the energy density. The stability analysis here is different from the previous theories [15] due to the additional gap energy of the pairing ground state. This condition is evaluated numerically in our calculation and the results have been shown in our main results below. Similar analysis for CDW and SDW phases can be also obtained [18].

In the numerical calculation of this paper, we consider two types of BFM systems for comparison: System A corresponds to the conventional ${ }^{40} \mathrm{~K}-{ }^{87} \mathrm{Rb}$ mixtures trapped in the optical lattices of a far-off resonant Nd:YAG laser and System B corresponds to the ${ }^{40} \mathrm{~K}-{ }^{23} \mathrm{Na}$ mixture loaded in the same trap. The interaction strength between different species of atoms can be tuned by either a relative shift of bosonic and fermionic lattice [20] or by tuning the system in the vicinity of interspecies Feshbach resonance [21]. If not specified, the interaction strengths used in this paper are referred to the background $s$-wave scattering lengths as listed in Ref. [14]. Other details about the lattice parameters are shown in the caption of Fig. 1.

In Fig. 1 we show the calculated many-body phase diagrams of both System A [in (a)] and System B [in (b)] as a function of fermion filling fraction $\left(n_{f}\right)$ and boson-fermion on-site interaction $\left(U_{b f}\right)$ in a regime where most competing orders can be observed. The phase boundaries are determined by comparing the mean-field $T_{c}$ of these many-body phases. From our numerical calculation, $T_{c}$ of the $s$-wave pairing phase of system $\mathrm{A}$ or the $d$-wave pairing of system B can be $1-3 \%$ of the Fermi energy $\left(=4 t_{f} \sim 1.53 \mathrm{kHz}\right)$ in the regime near half filling.

Several important results presented in Fig. 1 should be noted. First of all, SDW and CDW are dominant near the 


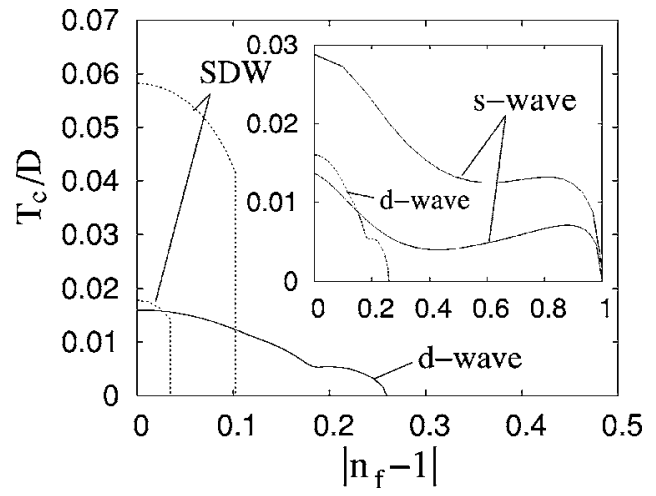

FIG. 2. Typical $T_{c}$ of the SDW phase and $d$-wave pairing phases as a function of filling fraction for system B (see text and Fig. 3 caption). $U_{f f} / E_{r}=0.52$ and 0.6 for the lower and upper curves of SDW phase, respectively. $D=4 t_{f}=0.34 E_{r}$ is half of the bandwidth. Inset: same but with $U_{f f} / E_{r}=0.32$ and 0.28 for the lower and upper curves of the $s$-wave pairing phase, respectively. $T_{c}$ of $p$-wave pairing phase is too small and not shown in this figure.

half-filling region due to the nesting effects (i.e., fermion filling is commensurate with the lattice spacing), and strong $U_{b f}$ favors CDW (or suppresses SDW) phase as shown in (a). This is because the phonon induced attractive interaction (proportional to $\left|U_{b f}\right|^{2} / U_{b}$ ) competes with the on-site repulsion between fermions. Second, the two density wave phases are separated by the superfluid phases, which is the usual $s$-wave pairing in system A but can be unconventional $(d$-or $p$-wave) pairing in system B. Physically, the unconventional pairing mechanisms arise when the boson correlation (healing) length is comparable to the lattice constant so that the strong intersite correlations are enhanced. In a typical ${ }^{40} \mathrm{~K}-{ }^{87} \mathrm{Rb}$ system shown in (a), such a requirement cannot be satisfied because the $\mathrm{Rb}$ atom is relatively heavy. As shown in (b) and in the later discussion, system B with lighter bosonic atoms $(\mathrm{Na})$ can have longer correlation length and therefore shows a wide regime for unconventional fermion pairing phases. Finally, within our mean-field analysis, a part of these unconventional pairing phases falls in the regime of collapse. However, we note that the mean-field approximation always strongly overestimates the collapse region, while correlation effects beyond the mean-field approximation can stabilize the uniform phases [22]. Thus Fig. 1(b) demonstrates that there is a wide parameter regime where one can observe unconventional fermion pairing in the K-Na BFM systems in a 2D optical lattice.

To study the competing orders further, in Fig. 2 we show the mean-field $T_{c}$ of the SDW and $d$-wave pairing phases for system B with two different values of $U_{f f}$. For strong on-site repulsive interaction, the SDW phase is favored near the half-filling $\left(n_{f}=1\right)$ regime because double occupancy of fermions is energetically unfavored and pseudospin Néel order is stabilized by the nesting effects. Away from half-filling, however, $d$-wave pairing becomes dominant because the usual $s$-wave pairing is suppressed by the strong on-site interaction. In the inset are the results for the same system but with even smaller $U_{f f}$, where the $s$-wave pairing phase becomes dominant as shown in Fig. 1(b). The $T_{c}$ of the $s$-wave pairing phase has a dip at $\left|n_{f}-1\right| \sim 0.5$ because the on-site

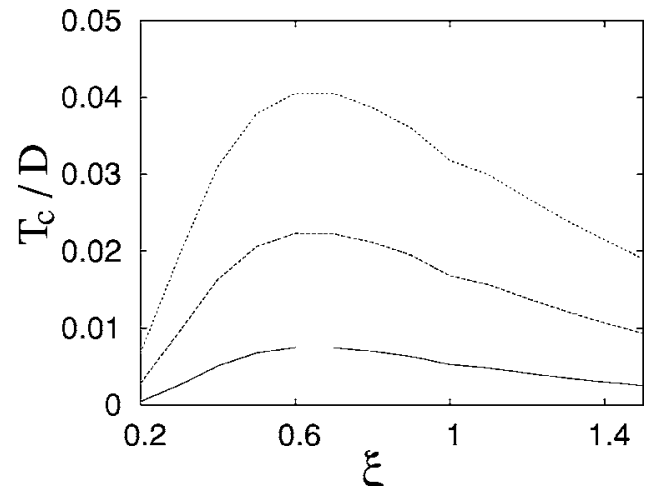

FIG. 3. $T_{c}$ of $d$-wave pairing phase at half-filling $\left(n_{f}=1\right)$ as a function of the healing length, $\xi$. Curves from bottom to up are for $\tilde{V} / E_{r}=0.53,0.88$, and 1.23 , respectively, and all the other parameters are the same as used for Fig. 2.

and the intersite components of $s$-wave pairing are dominant near half-filling and away from half-filling, respectively. From the typical value of $T_{c}$, we can estimate the effective BCS coupling strength $\lambda_{\mathrm{BCS}} \sim 0.4<1$. Therefore most of our results still fit reasonably well into a weak coupling regime where the mean-field approximation is justified.

To understand why System A (K-Rb mixture) does not provide unconventional pairing phases in the entire phase diagram [Fig. 1(a)], it is instructive to analyze how the $T_{c}$ of the $d$-wave pairing phase depends on the boson healing length $\xi$ in System B as shown in Fig. 3. Experimentally the healing length $\xi$ can be easily tuned by changing the density of bosonic atoms without affecting the stability of the whole system [15]. We find that for different values of phononinduced interaction strength $\widetilde{V}$, the maximum $T_{c}$ always corresponds to $\xi_{c} \sim 0.6$ (in the units of lattice constant), which is of the same order as lattice spacing for intersite pairing. However, for System A, the healing length of Rb is typically too short $(\xi \sim 0.12)$ to support intersite coupling. This explains why unconventional pairing phases are not present in system A and/or probably in most BFM systems with boson atoms heavier than fermions.

Low-temperature states of a BFM in the 2D optical lattice can be prepared by a process in which the sympathetic cooling of the atomic mixture is followed by an adiabatic cooling via increasing the lattice potential [23] until the phase transition is reached. Here we describe an alternative method to reach the low temperature unconventional pairing phases. Consider the situation in which a condensate mixture of bosonic atoms and molecules (composed by the fermions of two hyperfine states) is created. The lattice potential is then turned on and adiabatically increased such that the molecules are driven into a strongly localized state. A two-photon Raman pulse [24] is then applied to dissociate the bound molecule states into the lowest two-atom state in each lattice site. Finally, the lattice potential is reduced to the desired value such that the dissociated fermions begin to tunnel into neighboring sites while the bosons become condensed and then mediate the attractive interaction between fermions during an entire procedure. We point out that this method can be extremely efficient since the initial molecule condensate helps 
all Cooper pairs get closer to the zero momentum states and the relative motion of the pairing atoms after dissociation can be also kept in the lowest energy state if only the "melting" is slow enough. Finally, two-color photoassociation [26] can generate low lying Cooper pairs efficiently without heating [25], because both the molecules and the dissociated fermion pairs before melting are confined by a deep optical lattice and hence the energy cost for exciting to higher subbands can be much larger than the effective spontaneous rate.

The exotic many-body phases shown in Fig. 1 can be detected by various approaches due to their well-defined order parameters. First of all, CDW order can be observed in a standard time-of-flight measurement of bosons because the periodic density modulation of the boson condensate can be induced by the boson-fermion interaction and hence produces additional Bragg peaks at $\mathbf{Q}=(\pi, \pi)$. Besides, SDW order and the pairing phases can be further investigated by studying the noise correlation spectrum in the time-of-flight measurements [27]: The spectrum of pairing phase will have a sharp peak at zero momentum due to the condensation of Cooper pairs, while it will peak at momentum $\mathbf{Q}$ in the SDW phase due to the nesting effect. Furthermore, one can use Bragg scattering spectroscopy to probe the gap symmetry of the fermion pairing as proposed in Ref. [23]. For example, for the $d$-wave pairing phase a zero energy excitation should be observable when the momentum transfer of the two scattering photons matches any two of the nodal points on the Fermi surface. One can also use rf spectroscopy to measure the binding energy of fermion pairing [2] and the photoassociation method to study the superfluid fraction [26]. All these techniques have been used by a number of experimental groups.

In summary we investigated the many-body phase diagrams of a boson-fermion mixture in a $2 \mathrm{D}$ optical lattice. For a realistic ${ }^{40} \mathrm{~K}-{ }^{23} \mathrm{Na}$ or ${ }^{40} \mathrm{~K}-{ }^{87} \mathrm{Rb}$ system, a nature of superfluidity can be controlled by appropriate tuning of the atomic interaction strength and optical lattice parameters. Our results indicate that experimental studies of boson-fermion mixtures of cold atoms could have important implications for understanding the physics of unconventional superconducting materials.

We thank E. Altman, J. Fortagh, D. S. Petrov, and D. Podolsky for valuable discussions. This work was supported by the NSF (Grant Nos. DMR-01328074, PHY0134776), the Sloan and the Packard Foundations, and by Harvard-MIT CUA.
[1] G. Modugno et al., Science 297, 2240 (2002); J. Goldwin et al., Phys. Rev. A 70, 021601(R) (2004).

[2] C. Chin et al., Science 305, 1128 (2004).

[3] M. Greiner et al., Nature (London) 426, 537 (2003); S. Jochim et al., Science 302, 2101 (2003); M. W. Zwierlein et al., Phys. Rev. Lett. 91, 250401 (2003).

[4] M. Y. Kagan et al., Phys. Met. Metallogr. 99, 225 (2005); M. Lewenstein et al., Phys. Rev. Lett. 92, 050401 (2004); H. Fehrmann et al., Opt. Commun. 243, 23 (2004).

[5] R. Roth and K. Burnett, Phys. Rev. A 69, 021601(R) (2004); T. Miyakawa et al., cond-mat/0401107 (unpublished); H. P. Buchler and G. Blatter, Phys. Rev. Lett. 91, 130404 (2003); Phys. Rev. A 69, 063603 (2004).

[6] F. Matera, Phys. Rev. A 68, 043624 (2003); H. Heiselberg, et al., Phys. Rev. Lett. 85, 2418 (2000); L. Viverit, Phys. Rev. A 66, 023605 (2002).

[7] F. Illuminati and A. Albus, Phys. Rev. Lett. 93, 090406 (2004); A. Albus et al., Phys. Rev. A 68, 023606 (2003).

[8] L. Mathey et al., Phys. Rev. Lett. 93. 120404 (2004).

[9] We note that while there is no true long-range order in a $2 \mathrm{D}$ system at finite temperature due to thermal phase fluctuations, we expect the correlation length to increase rapidly below the mean-field $T_{c}$ and to become comparable to the system size for any realistic system.

[10] For a review see, D. J. Van Harlingen, Rev. Mod. Phys. 67, 515 (1995).

[11] See Organic Conductors: Fundamentals and Applications, edited by J. P. Farges (Marcel Dekker, New York, 1994), and reference therein.
[12] A. P. Mackenzie and Y. Maeno, Rev. Mod. Phys. 75, 657 (2003).

[13] D. Jaksch et al., Phys. Rev. Lett. 81, 3108 (1998).

[14] The background $s$-wave scattering lengths used in our calculation is given by $a_{\mathrm{K}-\mathrm{K}}=174, a_{\mathrm{Rb}-\mathrm{Rb}}=100, a_{\mathrm{K}-\mathrm{Rb}}=-330, a_{\mathrm{Na}-\mathrm{Na}}$ $=52$ and $a_{\mathrm{K}-\mathrm{Na}}=-129 a_{0}$, where $a_{0}$ is atomic unit.

[15] C. J. Pethick and H. Smith, Bose-Einstein Condensation in Dilute Gases (Cambridge, New York, 2002).

[16] J. R. Schrieffer, Theory of Superconductivity (Benjamin, New York, 1964).

[17] R. Micnas et al., Phys. Rev. B 37, 9410 (1988); Rev. Mod. Phys. 62, 113 (1990).

[18] D.-W. Wang, M. D. Lukin, and E. Demler, unpublished.

[19] E. W. Carlson, D. Orgad, S. A. Kivelson, and V. J. Emery, Phys. Rev. B 62, 3422 (2000); Jan Zaanen and Olle Gunnarsson, ibid. 40, R7391 (1989).

[20] O. Mandel et al., Phys. Rev. Lett. 91, 010407 (2003).

[21] H. Feshbach, Ann. Phys. 19, 287 (1962).

[22] A. P. Albus, F. Illuminati, and M. Wilkens, Phys. Rev. A 67, 063606 (2003).

[23] W. Hofstetter et al., Phys. Rev. Lett. 89, 220407 (2002).

[24] B. Deb and G. S. Agarwal, Phys. Rev. A 65, 063618 (2002); M. Mackie et al., Phys. Rev. Lett. 84, 3803 (2000).

[25] D. Jaksch et al., Phys. Rev. Lett. 89, 040402 (2002); T. Rom et al., ibid. 93, 073002 (2004).

[26] R. Wynar et al., Science 287, 1016 (2000).

[27] E. Altman et al., Phys. Rev. A 70, 013603 (2004); S. Folling et al., Nature (London) 434, 481 (2005). 